\title{
Time trend and age-period-cohort effects on gastric cancer incidence in Zaragoza and Navarre, Spain
}

\author{
N Aragonés, M Pollán M, G López-Abente, M Ruiz, A Vergara, C Moreno, P Moreo, \\ E Ardanaz
}

\begin{abstract}
Study objective-To describe time trends in gastric cancer incidence in Zaragoza and Navarre, and to investigate time period and birth cohort as determinants of such trends.

Design-Cases from two registries were grouped into five year intervals and the following were calculated: age specific and sex specific incidence rates, age adjusted incidence rates, cumulative rates, and the male to female ratio. Log linear models including age, period of diagnosis, and birth cohort were fitted.
\end{abstract}

Setting-The Zaragoza Cancer Registry covers the province of Zaragoza, which has a population of 824776 (403 755 men and 421021 women). The Navarre Cancer Registry covers the province of Navarre which has 512512 inhabitants (254 786 men and 257726 women). In both cases population figures were based on the 1986 census.

Patients-These comprised incident cases of gastric cancer reported to the Zaragoza Cancer Registry in 1963-87 and to the Navarre Cancer Registry in 1973-87.

Main results-Navarre registered higher adjusted and cumulative rates than Zaragoza for both sexes. In both provinces, there were relative declines in the rates for men and women of $3 \%$ and $4 \%$ respectively per year. In Zaragoza, the risk of developing stomach cancer fell in generations born between 1888 and 1933, and rose in subsequent birth cohorts in both sexes, while in Navarre the cohort effect showed an approximately linear decrease in risk for both sexes. Both provinces recorded increases in risk associated with cohorts born between 1933 and 1943.

Conclusions-The incidence rates of gastric cancer fell in both Zaragoza and Navarre. The reason for the greater incidence of gastric cancer in Navarre remains unknown. Trends in rates seem to be mainly linked to birth cohort. Increases in risk in generations born after 1933 may be ascribable to nutritional deficiencies in the early years of life.

\section{( $(\mathcal{E}$ Epidemiol Community Health 1997;51:412-417)}

The decline in gastric cancer incidence and mortality is a worldwide phenomenon coinciding with the second half of this century. ${ }^{12}$
Nevertheless, it is still one of the most frequent cancers in the world and occurs twice as frequently in men than in women. ${ }^{3}$

Gastric cancer exhibits noticeable international differences. European population based registries with the highest and lowest gastric cancer incidence rates for both sexes are St Petersburg, Russia (52.8 cases per 100000 person-years for men and 25.3 for women) and Tarn, France (8.3 for men and 3.4 for women), respectively. ${ }^{4}$ In Spain, gastric cancer not only registers high levels of incidence and mortality (above the European Community average ${ }^{45}$ ) but in 1985 was the second leading cause of cancer deaths in men and the third in women. ${ }^{6}$ As yet, no complete explanation has been forthcoming as to the important geographical differences that exist between and within countries or the factors associated with the development of this tumour.

Study of the effects of age, time period, and birth cohort may be helpful in gaining further insight into the causes of stomach cancer. This type of analysis has been carried out on the basis of incidence and mortality data in different countries. The existence of follow up data for a minimum period of 15 years at the Zaragoza and Navarre Cancer Registries has made it possible for such a study to be undertaken in Spain. Our study aimed to describe the time trends for gastric cancer incidence in Zaragoza and Navarre and to investigate time period and birth cohort as determinants of such trends.

\section{Methods}

CANCER REGISTRIES

The longest established cancer registries in Spain are those of Zaragoza (1960) and Navarre (1973), which together constitute the sole sources of incidence data going back more than 15 years. The Zaragoza Cancer Registry covers the province of Zaragoza which, based on the 1986 census, has a population of 824776 (403 755 males and 421021 females), while the Cancer Registry of Navarre covers the Autonomous Region of Navarre (in essence, a region comprising a single province), with 512512 inhabitants (254786 males and 257726 females). The geographical area covered by these two registries lies in the north of the Iberian Peninsula. Zaragoza is bordered to the west by the Autonomous Region of Navarre.

This study is based on incident cases of gastric cancer reported to the Zaragoza Cancer Registry in 1963-87, and the Navarre Cancer 
Table 1 Number of cases, age adjusted incidence rates per 100000 person years (European (E) and World (W) standard populations), and cumulative rate per 100 for the population aged 0-74 years in Zaragoza (1963-87) and Navarre (1973-87), Spain

\begin{tabular}{|c|c|c|c|c|c|c|c|c|c|}
\hline & \multicolumn{4}{|l|}{ Men } & \multicolumn{5}{|c|}{ Women } \\
\hline & Cases & $A R(E)$ & $A R(W)$ & $C R$ & Cases & $A R(E)$ & $A R(W)$ & $C R$ & $M: F$ \\
\hline \multicolumn{10}{|l|}{ Zaragoza } \\
\hline $1963-67$ & 761 & 53.93 & 36.59 & 4.47 & 598 & 34.13 & 22.14 & 2.61 & 1.58 \\
\hline $1968-72$ & 781 & 50.22 & 33.11 & 3.92 & 574 & 28.20 & 18.00 & 2.01 & 1.78 \\
\hline $1973-77$ & 726 & 41.62 & 27.44 & 3.27 & 561 & 24.15 & 15.12 & 1.63 & 1.72 \\
\hline $1978-82$ & 585 & 30.09 & 19.61 & 2.12 & 437 & 16.27 & 10.12 & 1.01 & 1.85 \\
\hline $1983-87$ & 666 & 31. & 21. & 2. & 423 & 14.54 & 9.52 & 1.04 & 2.16 \\
\hline \multicolumn{10}{|l|}{ Navarre } \\
\hline 1973-77 & 531 & 53.12 & 35.34 & 4.0 & 355 & 27.86 & 18.12 & 2.04 & 1.91 \\
\hline $1978-82$ & 536 & 47.31 & 31.98 & 3.83 & 313 & 21.20 & 13.66 & 1.45 & 2.23 \\
\hline 1983-87 & 477 & 38.58 & 25.68 & 2.83 & 295 & 18.00 & 11.83 & 1.26 & 2.14 \\
\hline
\end{tabular}

$\mathrm{AR}(\mathrm{E})$ European age adjusted incidence rate; $\mathrm{AR}(\mathrm{W})$ world age adjusted incidence rate; $\mathrm{CR}$ cumulative rate; M:F male to female ratio.

Registry in 1973-87. At the time the study was carried out, the last complete year available was 1987. Data on gastric cancer correspond to rubric 151 of the International Classification of Diseases (9th rev), and were extracted from the registers on a case by case basis. Data gathering methods employed by the two registries are: Zaragoza, mixed (active and passive): Navarre, active. A more detailed description of both registries is given elsewhere. ${ }^{47}$ Population estimates were calculated from all available censuses and municipal rolls for the study period using a log linear polynomial model. ${ }^{8}$

\section{STATISTICAL METHODS}

Age specific and sex specific incidence rates were calculated by grouping cases into five year intervals in both registries. Incidence rates were age adjusted by the direct method to the European and world standard populations, and, as an alternative to standardisation, cumulative rates for 0-74 years were also calculated. Relative risk was computed for male versus female patients.

To investigate the risk associated with specific generations, the number of cases in each group and period was assumed to constitute a variable with a Poisson distribution, and $\log$ linear models including age, period of diagnosis, and birth cohort were then fitted to the rates for each registry in both sexes. We originally studied the Zaragoza and Navarre registries jointly, using age-period-cohortregistry models including all possible interaction terms. However, in view of the existence of interactions between the registry term and the other variables, it was decided that a separate analysis would be presented for each registry.

Analysis was restricted to the population aged under 85 years, and the youngest group included in the model was the first group over the age of 14 years found to have at least five cases of gastric cancer in each quinquennium.

We first tested whether any age specific rates exhibited a degree of variability greater than that permitted by a Poisson distribution (over dispersion of rates). In such cases, the variance estimators from the Poisson model could lead to some factors proving statistically significant without really being so. Hence, where this
KEY POINTS

- In both Zaragoza and Navarre the incidence of gastric cancer fell between 1963 and 1987.

- The rates observed in young women may indicate a levelling off in incidence in the most recent generations.

proved to be the case, we proceeded to use a corrected model (gamma-Poisson model) which took over dispersion into account. ${ }^{9}$

The fact that a model which simultaneously considers age, period, and cohort has no single specific solution (non-identifiability problem), means that additional constraints have to be imposed. ${ }^{101112}$ We opted for one of the solutions described in published reports, whereby the overall slope of the period effect is assumed to be zero. ${ }^{1314}$ The rationale for this approach is that cohort has a stronger association with incidence than period..$^{15}$ Furthermore, exposure to risk factors associated with this tumour seems to be determined to a great extent by birth cohort. ${ }^{1618}$ Cohort and period of diagnosis values were averaged to unity.

\section{Results}

Table 1 shows the following: the absolute number of cases of gastric cancer diagnosed in Zaragoza (1963-87) and Navarre (1973-87) for men and women; age adjusted incidence rates per 100000 person-years (European and world standard populations); cumulative rates; and the male to female ratio. Figure 1 depicts age adjusted gastric cancer incidence rates (European standard population) for Zaragoza and Navarre in both sexes.

Stomach cancer incidence fell in Zaragoza and Navarre in both sexes over the time periods studied. In Zaragoza, 3519 cases of stomach cancer were diagnosed in men and 2593 in women in the period 1963-87. Furthermore, male and female rates respectively fell from 53.93 and 34.13 per 100000 person-years in 1963-67 (European standard population) to 31.34 and 14.54 in $1983-87$. In Navarre, 1544 and 963 cases respectively were diagnosed in men and women in 1973-87. Adjusted and

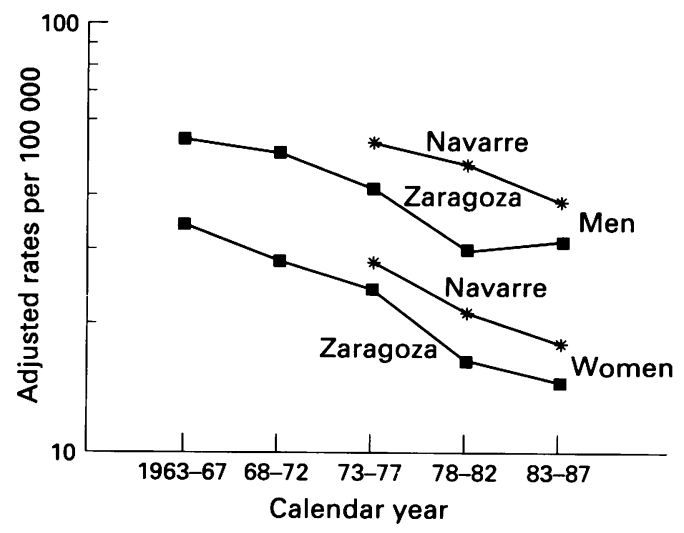

Figure 1 Age adjusted gastric cancer incidence rates by sex for Zaragoza and Navarre. 

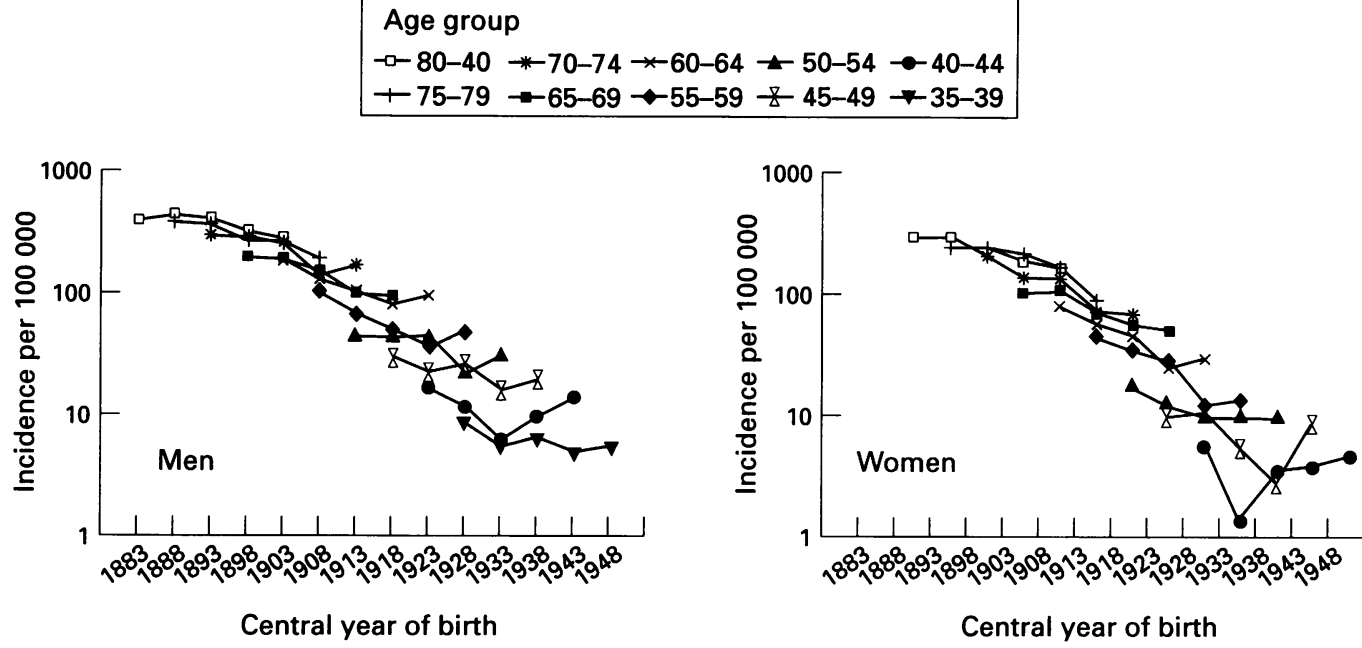

Figure 2 Age specific gastric cancer incidence rates by central year of birth and sex in Zaragoza.

Age group
$-\square-80-84 \rightarrow-70-74 * 60-64 \div 50-54 \rightarrow-40-44$
$+75-79 \rightarrow-65-69-55-59 \varangle 45-49 \rightarrow-35-39$
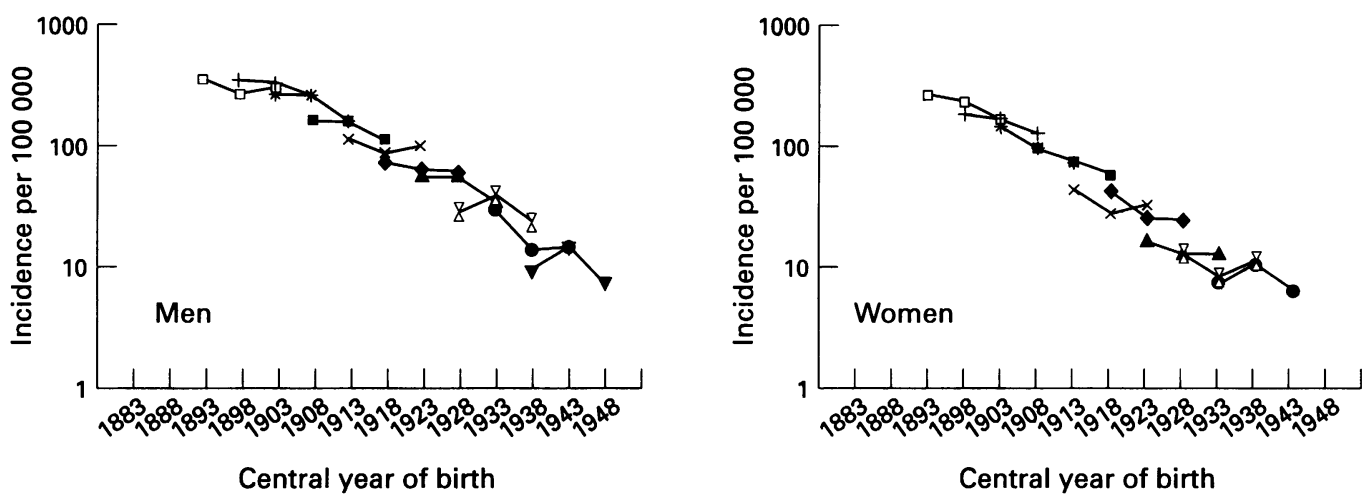

Figure 3 Age specific gastric cancer incidence rates by central year of birth and sex in Navarre.

cumulative rates for Navarre were higher than those for Zaragoza for both sexes. Indeed in men, the 1983-87 adjusted rates were almost as high as those reported for Zaragoza 10 years earlier. Both provinces showed overall relative declines in rates of $3 \%$ per year in men and $4 \%$ per year in women. However, as will be observed in figure 1 , there was a greater than expected fall off in male and female cases registered in Zaragoza between 1978 and 1982 . The overall male to female ratios were 1.9 in Zaragoza, with an almost constant upward rise throughout the study period, and 2.1 in Navarre.

Figures 2 and 3 plot age specific incidence rates for gastric cancer by median year of birth for male and female patients in Zaragoza and Navarre. In Zaragoza, rates fell for most age groups in both sexes during the penultimate five year period, with a subsequent rise thereafter in most age groups. In Navarre, while male trends were downward overall, for women aged under 65 , the trend ultimately levelled off.

Table 2 shows the goodness of fit of various Poisson models. The results from the two registries differed. In Zaragoza, the age + cohort model proved significantly better than age + drift model for men and women alike. Where men alone were concerned, however, the model which best fitted the data was age + period + cohort. In Navarre, age + drift models were found to adequately represent the data for both men and women.

Figure 4 shows the cohort and period effects resulting from the age + period + cohort models when the period slope is assumed to be equal

Table 2 Goodness of fit for different age, period, and cohort models of gastric cancer in Zaragoza and Navarre in both sexes

\begin{tabular}{|c|c|c|c|c|}
\hline \multirow[t]{2}{*}{ Models } & \multicolumn{2}{|l|}{ Men } & \multicolumn{2}{|l|}{ Women } \\
\hline & Deviance & $d f$ & Deviance & $d f$ \\
\hline \multicolumn{5}{|l|}{ Zaragoza } \\
\hline I Age & 208.2 & & 214.4 & \\
\hline II Age + drift & 57.9 & 39 & 46.6 & 35 \\
\hline III Age + period & 45.9 & 36 & 42.9 & 32 \\
\hline IV Age + cohort & 36.3 & 27 & 24.6 & 24 \\
\hline V Age + period + cohort & 24.3 & 24 & 21.6 & \\
\hline \multirow{2}{*}{\multicolumn{5}{|c|}{ Navarre }} \\
\hline & & & & \\
\hline $\begin{array}{l}\text { I Age } \\
\text { II Age + drift }\end{array}$ & $\begin{array}{l}28.7 \\
15.2\end{array}$ & $\begin{array}{l}20 \\
19\end{array}$ & $\begin{array}{r}37.3 \\
8.1\end{array}$ & $\begin{array}{l}18 \\
17\end{array}$ \\
\hline III Age + period & 14.3 & 18 & 8.0 & 16 \\
\hline IV Age + cohort & 8.9 & 9 & 4.9 & 8 \\
\hline V Age + period + cohort & 8.1 & 8 & 4.8 & 7 \\
\hline Overdispersion corrected & Yes & & No & \\
\hline
\end{tabular}

$\mathrm{df}=$ degrees of freedom 

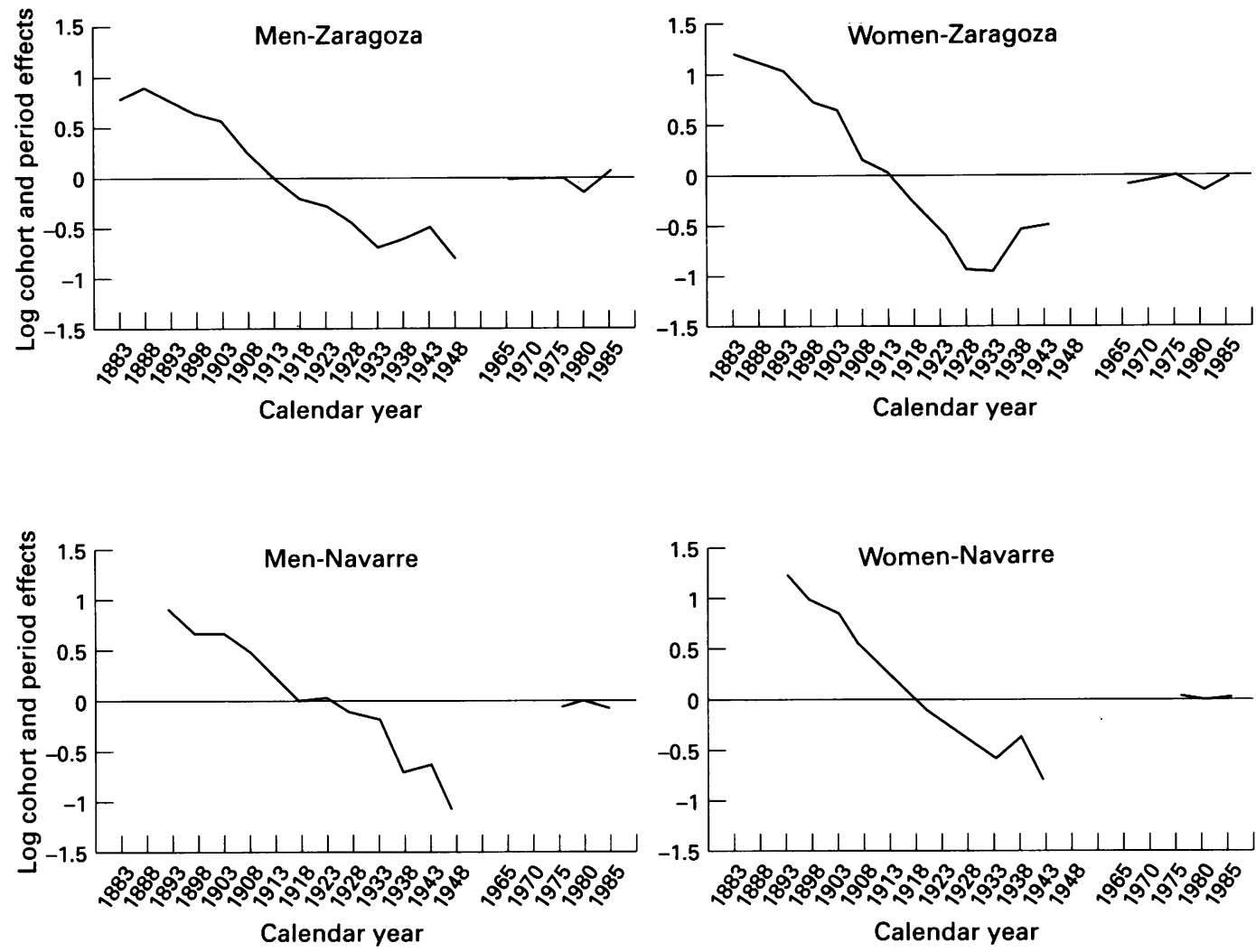

Figure 4 Cohort and period effect on gastrict cancer incidence by registry and sex. An overall period slope of zero was assumed.

to zero. Although the inclusion of the three factors is not necessary in all cases to explain the variability in the rates, the cohort and period effects of this model are depicted for greater ease of comparison between registries and sexes.

Taking the mean rate for all cohorts as reference, in Zaragoza the risk of developing stomach cancer declined in generations born between 1888 and 1933 and rose in subsequent birth cohorts in both sexes, though more noticeably so in women. In Navarre, the cohort effect showed an approximately linear decrease (on a logarithmic scale) in the risk of gastric cancer in both sexes for all generations studied, with fluctuations associated with certain birth cohorts. The patterns in Zaragoza and Navarre showed a peak in increased risk coinciding with the 1943 birth cohort in men and the 1938 birth cohort in women. The period effect in Zaragoza was affected by the 1978-82 decline in both sexes, which marked a departure from the general trend and was appreciable from observation of the adjusted and specific rates. The period effect in Navarre proved stable.

Net drift was $-0.141 /$ year for men and -0.188 for women in Zaragoza, amounting to a fall of $2.86 \%$ per year for men and one of $3.86 \%$ for women. In Navarre, net drift was -0.165 year in men and $-0.202 /$ year in women, which translates as respective falls of $3.36 \%$ and $4.12 \%$ per year in men and women.

Discussion

The results show that in both Zaragoza and Navarre the incidence rate of gastric cancer decreased. This decrease was more pronounced in women, leading to a rise in the male to female ratio over the study period. The trend in rates mainly seems to be related to birth cohort, as is borne out by the results from Zaragoza (the registry for which a longer follow up period is available). In Navarre, the rates observed in young women may indicate a levelling off in incidence in the most recent generations, something which remains to be confirmed in years to come.

The importance of studying the cohort effect resides in the fact that cohorts represent the most natural way of aggregating individuals, since the prevalence of aetiological factors linked to environment and/or lifestyle is to a great extent determined by year of birth. The use of age-period-cohort models implies the need to include additional assumptions. In this study the slope of the period effect was assumed to be equal to zero. Any improvement in the quality of registry data over time ${ }^{7}$ would be reflected in the model as an upward period effect. In this respect therefore, the above assumption tends to be conservative, in that the decline in incidence among the generations studied could be equal to or greater than that presented in this paper. This model shows that the risk of developing gastric cancer has declined in Zaragoza and Navarre, a phenomenon which, in overall terms, translates as a decrease in the rates of $3 \%$ per year in men and $4 \%$ in women. Despite this general trend, however, the downward movement is seen to halt in both sexes among post-1933 cohorts in Zaragoza, in line with mortality observations for this tumour nationwide. ${ }^{19}$ 
The substantial decline in gastric cancer in recent decades suggests that, in the main, environmental rather than genetic factors are implicated in its aetiology. ${ }^{16}$ Gastric cancer aetiology has been associated with dietary habits during the first years of life $\mathrm{e}^{1620}$ and Helicobacter pylori infection. ${ }^{2122}$ The rise in risk registered in Zaragoza for the most recent study cohorts - namely, those born during the Spanish Civil War and postwar period-may be linked to the dramatic situation prevailing in Spain at the time. Widespread scarcity had a considerable impact on diet and there was probably an increased prevalence of infection with Helicobacter pylori. In this respect and notwithstanding the fact that the downward trend is linear, in Navarre the cohort effect indeed shows specific rises in risk in both sexes among the war and postwar generations.

Trends in the appearance of gastric cancer have been variously described on the basis of histological type ${ }^{23}$ and subsite. ${ }^{17}$ While incidence of and mortality from adenocarcinoma of the gastric cardia have increased over recent decades, those for adenocarcinoma of the distal stomach have fallen. ${ }^{2324}$ Such disparity as well as the different epidemiologic characteristics, suggest that we are confronted with two aetiologically different entities. Incidence patterns in Zaragoza and Navarre could not, however, be analysed by anatomic subsite, since 4 th digit coding had not been widely enough applied to data at these cancer registries.

With respect to the period effect, the most noteworthy aspect is the fall observed in the 1978-82 period in Zaragoza. One underlying cause affecting data quality is incomplete case finding. In 1980, case finding, which until then had been passive, underwent a change in Zaragoza. Thenceforth, the role of voluntary reporters became limited to concrete rural areas, while active case searching and arrangements with hospital registries grew in importance. This new approach, though still not fully operational in the years immediately following its introduction, could have given rise to the subregister which seems to be present in the penultimate five year period. The day to day running and methodology of the Navarre Cancer Registry remained basically unchanged throughout the study period.

The incidence found in Navarre was higher than that of Zaragoza. The Navarre:Zaragoza incidence rates ratio ranged from 1.23 to 1.57 in men and from 1.15 to 1.3 in women for the period 1973-87. The better quality indices of the Navarre registry may point to the existence of a subregister in Zaragoza. Thus, the Zaragoza figures for all cases with histological confirmation were $67.0 \%$ in men and $50.8 \%$ in women, as against $86.2 \%$ and $76.9 \%$ respectively for Navarre, ${ }^{7}$ and the proportion of diagnoses ascertained via death certificates in Zaragoza totalled $19.8 \%$ in men and $29.6 \%$ in women versus $10.1 \%$ and $19.1 \%$ respectively in Navarre. ${ }^{7}$ Despite these differences, the existence of a real difference in tumour incidence as between these two provinces cannot be ruled out. Geographical differences have been linked to environmental factors more common in rural settings, such as high consumption of sausage meats (meats which tend, moreover, to be preserved using traditional methods). In this respect, it should be stressed that Navarre has a larger rural population $(40 \%$ of the population live in towns with under 5000 inhabitants, and only $36 \%$ live in the provincial capital, Pamplona), ${ }^{4}$ whereas in the province of Zaragoza, $75 \%$ of the population live in the capital. ${ }^{26}$

Gastric cancer mortality rates for Navarre are likewise higher than those for Zaragoza, and both are higher than elsewhere in Spain. Spanish age adjusted mortality rates (standard European population) for the periods $1972-76$ and $1982-86$ respectively were 38.81 and 25.03 per 100000 person-years in men and 21.22 and 12.44 in women. ${ }^{19}$ In spite of the differences between mortality and incidence data, the decline in gastric cancer incidence in Zaragoza and Navarre parallels the decline in gastric cancer mortality nationwide. ${ }^{19}$ The fact that these trends are the same may indicate that, overall, there have been no major changes in survival or case registration.

The Spanish cancer registries reporting the highest gastric cancer rates are those of Navarre, Zaragoza, and the Basque Country. ${ }^{4}$ The rates for Zaragoza and Navarre are high compared with those for the European Union (EU). Expressed in concrete terms, Navarre's rates rank only behind those quoted for the following registries: Vila Nova de Gaia (Portugal); Florence, Lombardy Region (Varese Province), Parma Province and Romagna (Italy); and the Basque Country (Spain). In women, the Navarre registry rates are solely exceeded by those of the above Portuguese and Italian registries. On the other hand, the decline in incidence rates in Spain is similar to that observed for the remaining EU member states. ${ }^{1}$

This study describes the decline in gastric cancer incidence for both sexes as observed in Zaragoza and Navarre over recent decades. The higher incidence of gastric cancer in Navarre may be due to greater exposure to risk factors for stomach cancer, the better quality of the data on its register, or a combination of the two. Age-period-cohort analyses furnish evidence of increased risk in certain birth cohorts, possibly associated with rises in exposure to risk factors. Bearing in mind that other studies have suggested a possible future levelling off in rates in developed countries, ${ }^{1727}$ a longer period of observation is required for ascertainment of the trend -downward at present- in the incidence of this tumour in years to come.

Funding: this study was supported by the Health Research Funding: this study was supported by the Health Research Sanitaria grant $94 / 150$ Sanitaria, grant $94 / 150)$.

1 Coleman MP, Estève J, Damiecki P, Arslan A, Renard H. Trends in cancer incidence and mortality. IARC Scientific Trends in cancer incidence and mortality. IARC Scientific Publications No 121 . Lyon

2 Geddes M, Balzi D, Tomatis L. Progress in the fight against cancer in EC countries: changes in mortality rates, 1970 90. Eur f Cancer Prev 1994;3:31-44.

3 Parkin DM, Läärä E, Muir CS. Estimates of the worldwide frequency of sixteen major cancers in 1980. Int $\mathcal{F}$ Cancer 1988;41:184-97. 
4 Parkin DM, Muir CS, Whelan SL, Gao YT, Ferlay J, Powell J. Cancer incidence in five continents. Vol VI. IARC Scientific Publications No 120. Lyon: International Agency for Research on Cancer, 1992.

5 Jensen OM, Esteve J, Møller H, Renard H. Cancer in the European Community and its member states. Eur $\mathcal{F}$ Cancer 1990; 26:1167-256.

6 Bolumar F, Vioque J, Cayuela A. Changing mortality patterns for major cancers in Spain, 1951-1985. Int f Epidemiol 1991;20:20-25.

7 González J, Gorgojo L, Martín J, Villar F. Cancer en España. Madrid: Ministerio de Sanidad y Consumo, 1993.

8 Aickin M, Dunn CN, Flood TJ. Estimation of population denominators for public health studies at the tract, gender
and age-specific level. Am $\mathcal{F}$ Public Health 1991;81:918-20.

9 Breslow N. Extra-Poisson variation in log-linear models. Applied Statistics 1984;33:38-44.

10 Osmond C, Gardner MJ. Age, period and cohort models applied to cancer mortality rates. Stat Med 1982;1:245-59.

11 Decarli A, La Vecchia C. Age, period and cohort models: Decarli A, La Vecchia C. Age, period and cohort models:
review of knowledge and implementation in GLIM. Revista di Statistica Applicata 1987;20:397-410

12 Holford TR. Understanding the effects of age, period, and cohort on incidence and mortality rates. Annu Rev Public Health 1991;12:425-57.

13 Dubrow R, Bernstein J, Holford TR. Age-period-cohort modelling of large-bowel-cancer incidence by anatomic sub-site and sex in Connecticut. Int 7 Cancer 1993;53: 907-13.

14 Dubrow R, Johansen C, Skov T, Holford TR. Age-periodcohort modelling of large-bowel-cancer incidence by anatomic sub-site and sex in Denmark. Int $\mathcal{f}$ Cancer 1994;58: tomic sub-

15 Holford TR. Analysing the temporal effects of age, period and cohort. Stat Methods Med Res 1992;1:317-37.

16 Howson C, Hiyama T, Wynder E. The decline in gastric cancer: Epidemiology of an unplanned triumph. Epidemiol Rev 1986;8:1-27.

17 Zheng T, Mayne ST, Holford TR, et al. The time trend and age-period-cohort effects on incidence of adenocarcinoma of the stomach in Connecticut from 19551989. Cancer 1993;72:330-40.

18 Hansson LE, Bergström R, Sparen P, Adami HO. The decline in the incidence of stomach cancer in Sweden 1960-1984: a birth cohort phenomenon. Int $\mathcal{f}$ Cancer 1991;47:499-503.

19 López-Abente G, Pollán $M$, Ruiz $M$, Jiménez $M$, Vázquez F. Cancer mortality in Spain, 1952-1986. Effect of age, birth cohort and period of death. Madrid: Centro Nacional de Epidemiologia, 1992.

20 Correa P, Haenszel W, Cuello C, Tannenbaum S, Archer M. A model for gastric cancer. Lancet 1975;2:58-60.

21 Taylor DN, Blaser MJ. The epidemiology of Helicobacter pylori Infection. Epidemiol Rev 1991;13:42-59.

22 The Eurogast Study Group. An international association between Helicobacter pylori infection and gastric cancer. Lancet 1993;341:1359-62.

23 Laurén PA, Nevalainen TJ. Epidemiology of intestinal and diffuse types of gastric carcinoma. Cancer 1993;71:2926-33.

24 Craanen ME, Dekker W, Blok P, Ferwerda J, Tygat GNJ. Time trends in gastric carcinoma: changing patterns of type and location. Am f Gastroenterol 1992;87:572-79.

25 Blot WJ, Devesa SS, Kneller RW, Fraumeni JF. Rising incidence of adenocarcinoma of the esophagus and gastric cardia. $¥ A M A$ 1991;265:1287-89.

26 Vergara A, Moreo P, Guimbao J, Lázaro MA Incidencia de cáncer en Zaragoza 1983-1986. Resultados del Registro de Cáncer de Zaragoza. Zaragoza: Departamento de Sanidad y Concer de Zaragoza. Zaragoza: Departame. Gobierno de Aragón, 1993.

27 Capocaccia R, De Angelis R, Frova L, Sant M, Buiatti E, Gatta E. Estimation and projections of stomach cancer trends in Italy. Cancer Causes Control 1995;6:339-46. 